\title{
Identification of the ionizing source of NGC $2024^{\star}$
}

\author{
A. Bik ${ }^{1}$, A. Lenorzer ${ }^{1}$, L. Kaper ${ }^{1}$, F. Comerón ${ }^{2}$, L. B. F. M. Waters ${ }^{1,3}$, A. de Koter ${ }^{1}$, and M. M. Hanson ${ }^{4}$ \\ 1 Astronomical Institute “Anton Pannekoek”, University of Amsterdam, Kruislaan 403, 1098 SJ Amsterdam, \\ The Netherlands \\ 2 European Southern Observatory, Karl-Schwarzschild Strasse 2, Garching-bei-München, 85748, Germany \\ 3 Instituut voor Sterrenkunde, Katholieke Universiteit Leuven, Celestijnenlaan 200B, 3001 Heverlee, Belgium \\ ${ }^{4}$ University of Cincinnati, Cincinnati, OH 45221-0011, USA
}

Received 16 September 2002 / Accepted 21 February 2003

\begin{abstract}
We propose the late-O, early-B star IRS2b as the ionizing source of the Flame Nebula (NGC 2024). It has been clear that such a hot, massive star must be present in this heavily obscured region, and now it has been identified. New nearinfrared photometry shows that IRS2b is the most luminous and hottest star in the young star cluster embedded in the center of NGC 2024. The near-infrared observations $\left(5^{\prime} \times 5^{\prime}\right)$ cover $\sim 90 \%$ of the $\mathrm{H}$ II region detected in radio continuum radiation, making the probability very low that the ionizing star is not present in the field. A $K$-band spectrum of IRS2b obtained with ISAAC on the Very Large Telescope indicates that the spectral type of IRS2b is in the range O8 V - B2 V. Additional arguments indicate that its spectral type is likely closer to $\mathrm{O} 8$ than to $\mathrm{B} 2$. The corresponding amount of ionizing radiation is consistent with published radio continuum and recombination line observations.
\end{abstract}

Key words. stars: early-type - ISM: HII regions - ISM: individual objects: NGC 2024 - infrared: stars

\section{Introduction}

Just east of $\zeta$ Ori and north-east of the Horsehead Nebula, NGC 2024 (Flame Nebula) appears in visible light as a bright nebula of which the central part is obscured by a thick dust lane. The central region of NGC 2024 is also a bright source of radio continuum emission and recombination lines (Krügel et al. 1982; Barnes et al. 1989), indicating the presence of an ionizing star of spectral type 09-09.5. The heavy obscuration by dust is the reason why, contrary to M42 (O'dell 2001) and many other visible $\mathrm{H}$ II regions, the exciting star(s) of NGC 2024 have not been identified. Near- and mid-infrared observations have revealed the presence of a young star cluster in the core of NGC 2024 (Barnes et al. 1989; Lada et al. 1991; Comerón et al. 1996; Haisch et al. 2001). Several candidates for the ionizing source of NGC 2024 have been proposed (Grasdalen 1974; Barnes et al. 1989), but none of these candidates are able to produce the observed radio continuum emission.

We are carrying out a systematic survey of the stellar content of compact and ultra-compact H II regions, with the aim to detect and study deeply embedded, newly-born massive stars. Our ultimate goal is to better understand the earliest phases in the life of the most massive stars. In this context we have obtained deep near-infrared images of a sample

Send offprint requests to: A. Bik, e-mail: bik@science.uva.nl

* Based on observations collected at the European Southern Observatory at La Silla and Paranal, Chile (ESO programmes 62.H-0443 and 64.H-0425). of compact H II regions, including NGC 2024 (Kaper et al. 2003). Subsequent $K$-band spectroscopy of the candidate ionizing stars has resulted in the determination of their spectral types (Bik et al. 2003).

Here we describe how we have identified the long-soughtfor ionizing star of NGC 2024. In Sect. 2 we present nearinfrared, narrow-band images of this region, as well as a $K$-band spectrum of the candidate ionizing star IRS2b. In Sect. 3 we derive the physical properties of this star. In the last section we present our conclusions and compare the derived properties of IRS2b with alternative, previously proposed candidates.

\section{Observations}

Near-infrared images of the central region of NGC 2024 were obtained with SOFI mounted on ESO's New Technology Telescope (NTT) at La Silla on February 6, 1999; the seeing was $0.7^{\prime \prime}$. We used narrow-band filters centered on strong nebular emission lines ( $\left.\mathrm{P} \beta 1.28 \mu \mathrm{m}, \mathrm{H}_{2} 2.12 \mu \mathrm{m}, \mathrm{Br} \gamma 2.16 \mu \mathrm{m}\right)$, and two narrow-band continuum filters in the $J$ and $K$-band $(1.21$ and $2.09 \mu \mathrm{m})$. The latter were chosen to measure the $J$ and $K$ magnitude of the embedded stars, avoiding the contamination by nebular emission lines.

Nine frames of $20 \mathrm{~s}$ each were taken on source and 9 frames with a $2 \mathrm{~s}$ exposure per frame were obtained to measure the sky background. The observations were reduced using standard infrared imaging data reduction procedures with IRAF. 
Pointsources were detected by adding the frames in the $J$ and $K$ continuum filters, and running DAOPHOT (Stetson 1987) on the resulting frame. The photometry was obtained as follows: aperture photometry with a large aperture was performed on the image of the standard star and of bright, isolated stars in the image field. This allowed us to set up a network of bright secondary standards in the image field. Then, aperture photometry with a small aperture (3 pixel in radius), adequate for our rather crowded field, was performed on all the stars in the field. The magnitudes of these stars were determined taking those of the secondary standards as a reference.

We note that for red sources $((J-K) \gtrsim 2 \mathrm{mag})$ the narrowband $J$ and $K$ magnitudes differ significantly from the broadband $J$ and $K_{\mathrm{s}}$ magnitudes. This difference has been estimated by multiplying various energy distributions with the response curves of the filters, representing a range in $(J-K)$ from 0 to 7 . The typical errors for $(J-K) \approx 5$ on this correction are 0.2 and 0.07 mag in $J$ and $K$, respectively. The $J$ and $K$-band magnitudes used in this paper are based on narrow-band observations. Only for the construction of the Hertzsprung-Russell diagram (HRD, Fig. 4) the narrow-band magnitudes are converted into broad-band magnitudes.

Medium-resolution $(R \simeq 8000) K$-band spectra of some stars in this region were taken with ISAAC and ESO's Very Large Telescope (VLT) at Paranal, Chile, on March 20, 2000. The spectra were dark- and flat-field corrected, and wavelength calibrated using standard reduction procedures. In order to correct for the sky background the object was "nodded" between two positions on the slit (A and B) such that the background emission registered at position $\mathrm{B}$ (when the source is at position A) is subtracted from the source plus sky background observations at position $\mathrm{B}$ in the next frame, and vice versa. Telluric absorption lines were removed using the telluric standard star HD 39908 with spectral type A2 V observed under identical sky conditions. The only photospheric line $(\mathrm{Br} \gamma)$ in the spectrum of the telluric star needs to be divided out first. It turns out that the best result is achieved when first the telluric features are removed from the $K$-band spectrum of the telluric standard using a high resolution telluric spectrum (obtained by $\mathrm{NSO} /$ Kitt Peak). This spectrum is taken under completely different sky conditions, so a lot of remnants are still visible in the corrected standard star spectrum; without this "first-order" telluric correction, a proper fit of $\mathrm{Br} \gamma$ cannot be obtained. The $\mathrm{Br} \gamma$ line is fitted by a combination of two exponential functions. The resulting error on the $\mathrm{Br} \gamma$ equivalent width $(\mathrm{EW})$ of our target star is about $5 \%$.

An $L$-band spectrum of IRS2b, with a spectral resolution $R \simeq 1200$ was obtained with VLT/ISAAC on February 22, 2002. The data reduction was performed using an A6 II star (HD 73634) as telluric standard. The quality of the telluric standard was not sufficient to obtain a quantitative measurement of the hydrogen absorption lines in the $L$ band spectrum.

\section{The nature of the ionizing source in NGC 2024}

Figure 1 shows a composite near-infrared image of the obscured central region of NGC $2024\left(5^{\prime} \times 5^{\prime}\right)$, centered on the bright (saturated, $K \sim 5$ ) infrared source IRS2. Exposures in three narrow-band filters are combined: $\mathrm{Br} \gamma, \mathrm{H}_{2}$ and $\mathrm{P} \beta$. The edge of the molecular cloud to the south of NGC 2024 is marked by emission produced by excited molecular hydrogen: note the clumpy filamentary structure. The recombination lines of hydrogen (e.g. Br $\gamma$ ) are strongest towards the core of the embedded stellar cluster. The optical extent of the Flame Nebula is roughly $15^{\prime} \times 15^{\prime}$, but at radio wavelengths NGC 2024 is only slightly larger $\left(6^{\prime} \times 6^{\prime}\right)$ than the field covered by our near-infrared observations. The radio continuum radiation is strongly concentrated towards the central part of NGC 2024, and is expected to outline the extent of the region where hydrogen is fully ionized.

In the following we demonstrate that IRS2b is the best candidate ionizing star present in the central star cluster of NGC 2024. A $K$-band spectrum of IRS2b is used to confirm its early spectral type. The resulting effective temperature and luminosity of IRS2b are consistent with the amount of observed radio continuum and line emission.

\subsection{The young stellar cluster embedded in NGC 2024}

Due to the severe obscuration by the dust lane in front of NGC 2024, its stellar population becomes apparent at nearinfrared wavelengths. The strong infrared sources IRS1-5 are the brightest objects in Fig. 1. Another bright source, IRS2b is located just 5" north-west of IRS2 (see inset Fig. 1). The existence of IRS2b was first reported by Jiang et al. (1984) and confirmed by Nisini et al. (1994).

We detect 106 and 210 point sources down to the detection limit of $17.7 \mathrm{mag}$ in $J$ and of $16.1 \mathrm{mag}$ in $K$, respectively. Because of the proximity of NGC 2024 (360 pc, Brown et al. 1994), its small spatial extent, its angular separation from the galactic plane, and the obscuration of background sources by the molecular cloud, we expect that nearly all of the infrared sources present in our near-infrared image are physically related to the cluster (cf. Comerón et al. 1996).

The brightest infrared source in the center of the NGC 2024 cluster is IRS2, a source that has been held responsible for the ionization of the $\mathrm{H}$ II region since its discovery (Grasdalen 1974). However, the physical nature of IRS2 is unclear: its $K$ band magnitude is too bright for a main sequence star, indicating a strong infrared excess. This excess is likely due to the presence of a dense circumstellar disk; the central star is probably a B-type star (Lenorzer et al. 2003, and below). Can we identify another, potentially hotter star that could be responsible for the ionization of the Flame Nebula?

The $K$ vs. $(J-K)$ color-magnitude diagram (CMD) of the stars detected in our images is presented in Fig. 2; only the brightest objects are shown. The dotted line indicates the position of the main sequence (Aller et al. 1982), for different amounts of visual extinction $A_{V}$. A distance modulus of $7.80 \pm 0.45 \mathrm{mag}$ is adopted, which corresponds to $363 \pm 75 \mathrm{pc}$ (Brown et al. 1994). An anomalous extinction law with $R_{V}=$ 5.5 is used, as measured by Lee (1968). We used the parameterization of Cardelli et al. (1989) to describe the shape of the extinction law. This extinction law is derived for stars with $E(B-V) \leq 1$ and could be different for higher amounts 


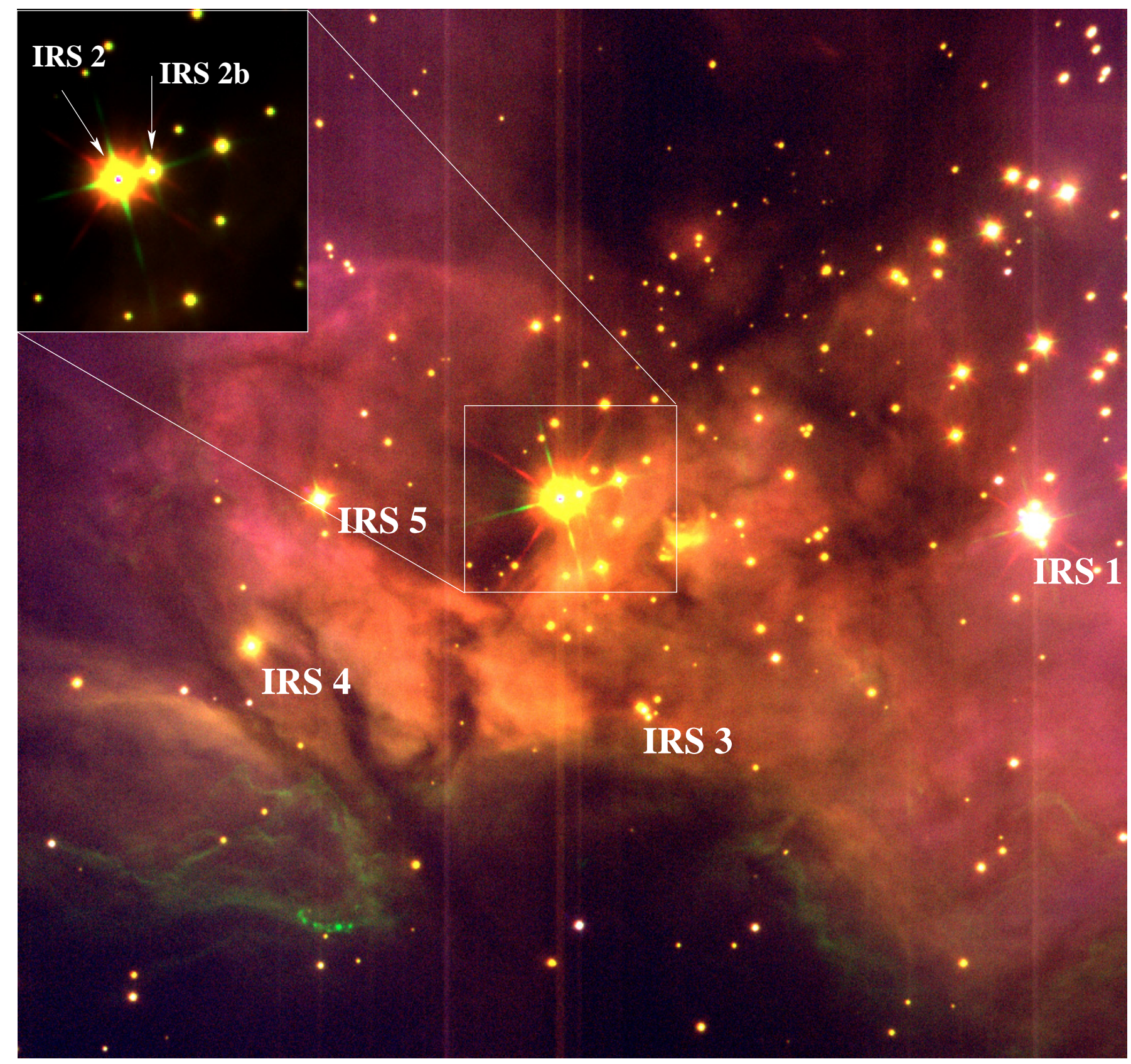

Fig. 1. Composite of three narrow-band images of the central region of NGC 2024 obtained with NTT/SOFI (blue: $\mathrm{P} \beta$; red: Br $\gamma$; green: $\mathrm{H}_{2}$ ). North is up and East to the left; the image size is $5^{\prime} \times 5^{\prime}$ (at the distance of NGC2024 this corresponds to $0.5 \times 0.5 \mathrm{pc}$ ). The upper left panel zooms in on the region around IRS2: about 5" to the north-west we find IRS2b, the ionizing star of NGC 2024. Also some previously proposed candidate ionizing stars are indicated. The vertical stripes are instrumental artifacts caused by the brightest stars.

of reddening. However, the dereddening of $\mathrm{OB}$ stars in the near infrared is not very sensitive to the precise value of $R_{V}$, as $A_{K}=0.108 \times A_{V}$ for $R_{V}=3.1$ and $A_{K}=0.125 \times A_{V}$ for $R_{V}=5.5$. The slope of the near-infrared extinction law changes very slowly with $R_{V}$ and the intrinsic $(J-K)$ color of OB stars is almost constant with spectral type and luminosity class (Koornneef 1983).

Figure 2 shows that the star IRS2b $(K=7.57 \pm 0.07)$, when dereddened to the main sequence, is the best candidate ionizing star in the field (neglecting IRS2) at a position consistent with a late $\mathrm{O}$ main sequence star. The position of IRS2b in the CMD implies $A_{V}=24.0 \pm 0.5 \mathrm{mag}\left(A_{V}=28.5 \pm 0.5\right.$ for $\left.R_{V}=3.1\right)$ if it is a main sequence star.

\subsection{K-band spectral type of IRS2b}

The spectral type of IRS2b can be determined from its $K$-band spectrum (Fig. 3). Hanson et al. (1996) define five $K$-band spectral classes for $\mathrm{O}$ and early-B stars based on low-resolution $K$-band spectra. Two $K$-band spectral classes correspond to the hottest $\mathrm{O}$ stars $(\mathrm{kO} 3-\mathrm{O} 4$ and $\mathrm{kO5-O6}$, with $\mathrm{k}$ denoting that the classification is based on the $K$-band spectrum) which 


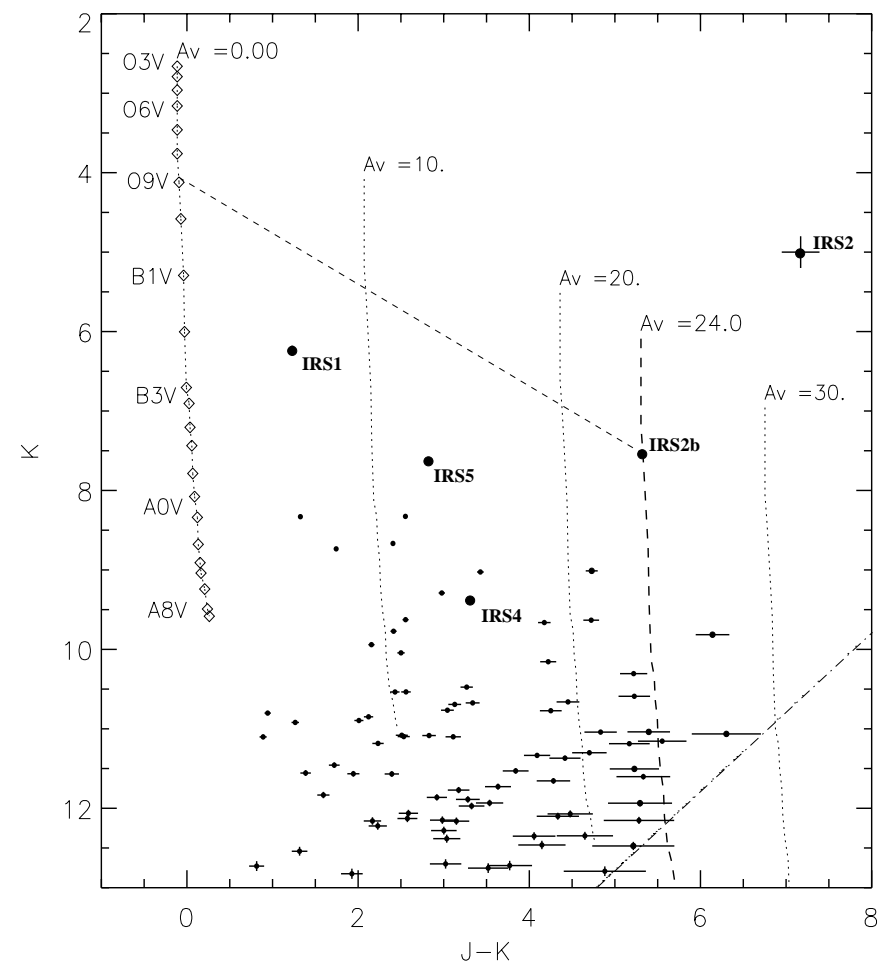

Fig. 2. Color-magnitude diagram of the brightest stars in the central region of NGC 2024. The vertical (dotted) lines indicate the position of the main sequence for different amounts of visual extinction $\left(A_{V}\right)$. The magnitudes are obtained through narrow-band observations. The diagonal (dashed) line is the dereddening line of IRS2b, demonstrating that IRS2 $b$ is the intrinsically brightest star of the embedded population. IRS2 (saturated in $K$ ) probably is a B star with a strong infrared excess due to a circumstellar disk (Lenorzer et al. 2003). The dasheddotted line is the detection limit in $(J-K)$ following from the detection limits in $J$ and $K$.

show lines of N III $(2.115 \mu \mathrm{m})$ and C IV $(2.079 \mu \mathrm{m})$ in emission and the $\operatorname{Br} \gamma(2.166 \mu \mathrm{m})$ line in absorption. In the third class (kO7-O8) the C IV line is absent and the He I (2.1128, $21137 \mu \mathrm{m})$ lines appear in absorption. The fourth spectral class kO9-B1 is defined as having $\mathrm{He} \mathrm{I}$ and $\mathrm{Br} \gamma$ both in absorption, with the equivalent width of $\mathrm{Br} \gamma$ less than about $4 \AA$. This class is equivalent to a Morgan-Keenan (MK) spectral type between $\mathrm{O} 8 \mathrm{~V}$ and B1 V. The fifth class (kB2-B3) shows strong Br $\gamma$ and $\mathrm{He} \mathrm{I}$ absorption lines and is equivalent to MK type B1 V to B2 V. The line equivalent widths (EW) can in principle be used to determine the $K$-band spectral type. There is, however, a substantial scatter in the observed EW of $\mathrm{Br} \gamma$ as a function of spectral type in the calibration stars used by Hanson et al. (1996).

IRS2b has both He I $2.113 \mu \mathrm{m}$ and $\mathrm{Br} \gamma$ in absorption, with an equivalent width of $0.4 \pm 0.1 \AA$ and $4.9 \pm 0.6 \AA$, respectively. The $\mathrm{Br} \gamma \mathrm{EW}$ indicates that the $K$-band spectral type of IRS2b is in the range $\mathrm{kO} 9-\mathrm{B} 1$ to $\mathrm{kB} 2-\mathrm{B} 3$ (MK types O8-B2). The He I line appears in absorption around spectral type O7.5, increasing its EW towards later spectral types. The He I line decreases in strength again towards B2/B3. The relatively weak He I line in the spectrum of IRS2b suggests that its spectral type is either close to $\mathrm{O} 8$, or more towards $\mathrm{B} 2 / \mathrm{B} 3$.

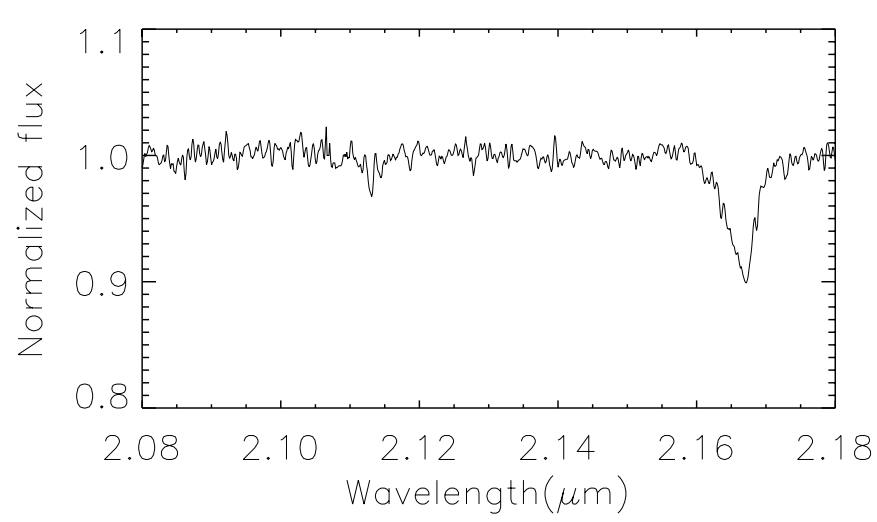

Fig. 3. $K$-band spectrum of IRS2b, obtained with VLT/ISAAC, showing He I 2.1127, $2.1138 \mu \mathrm{m}$ and $\mathrm{Br} \gamma 2.166 \mu \mathrm{m}$ in absorption.

An $L$-band spectrum provides a better diagnostic of its spectral type, since it includes hydrogen Pfund lines, which are more temperature sensitive than $\operatorname{Br} \gamma$ (Lenorzer et al. 2002). The $L$-band spectrum we obtained from IRS2b is of insufficient quality to measure the strength of the absorption lines, though it is clear that they are present. Although we are not able to provide an independent estimate of its spectral type, the $L$-band spectrum rules out the possibility that IRS2b has an infrared excess. Such an excess would be even more dominant in the $L$-band, hindering the detection of photospheric lines.

\subsection{The position of IRS2b in the HRD}

Taking our measurement errors into account, we arrive at a (conservative) estimate of the MK spectral type of IRS2b in the range between $\mathrm{O} 8 \mathrm{~V}$ and $\mathrm{B} 2 \mathrm{~V}$. This corresponds to a range in $T_{\text {eff }}$ between 34000 and $22000 \mathrm{~K}$ (Martins et al. 2002; Aller et al. 1982). Note, however, that the effective temperature calibration of OB stars is still a matter of debate. The derived limits on $T_{\text {eff }}$ are not very hard.

For every $T_{\text {eff }}$, the dereddened $K$-band magnitude can be converted into a luminosity (adopting a distance of $363 \mathrm{pc}$ ). For this calculation we use the relation between the bolometric correction and $T_{\text {eff }}$ from Vacca et al. (1996) and the $(V-K)$ vs. $T_{\text {eff }}$ relation given by Koornneef (1983). The uncertainty in the luminosity of IRS2b is due to the uncertainty in the distance towards NGC 2024 and to the conversion from narrowband to broad-band magnitudes. The observational constraints on the location of IRS2b in the HRD are visualized in Fig. 4 (left panel, shaded area).

Are these constraints on $L$ and $T_{\text {eff }}$ of IRS2b consistent with the observed nebular emission? The radio continuum flux of the $\mathrm{H}$ II region can be used to estimate the ultraviolet flux of the ionizing source of NGC 2024. The total radio continuum flux at $1667 \mathrm{MHz}$ derived from high-resolution radio observations (Barnes et al. 1989) is $63 \pm 4$ Jy. The number of Lyman continuum photons is derived from the observed radio continuum flux under the assumption that the $\mathrm{H}$ II region is ionization bounded and neglecting absorption of UV radiation by dust, implying that the number of recombinations is equal to the number of 

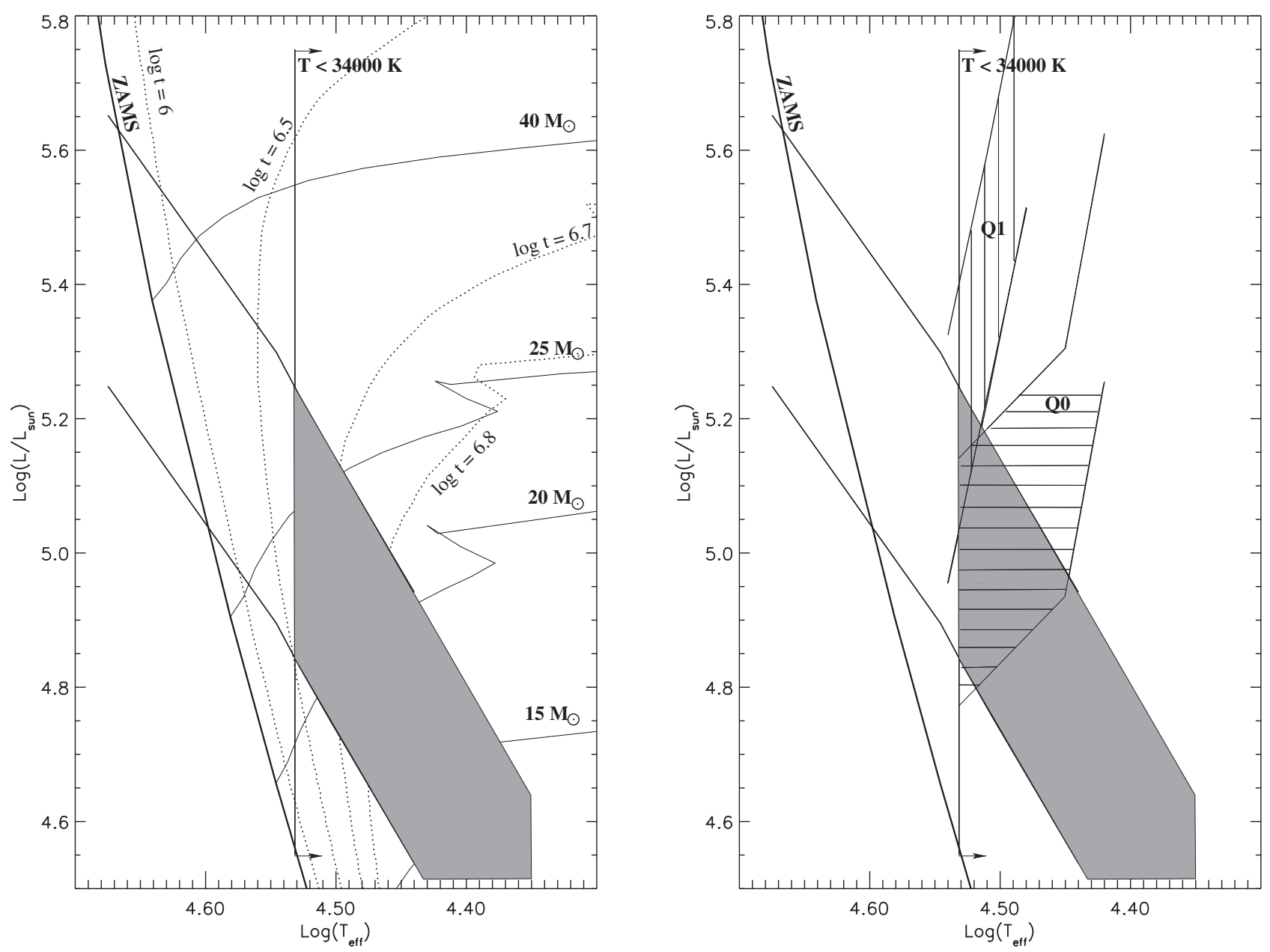

Fig. 4. Left: the upper part of the theoretical HRD. The shaded region indicates the position of IRS2b for which all observational constraints on $L$ and $T_{\text {eff }}$ are fulfilled. The diagonal area represents the constraint provided by the observed $K$-band magnitude. The $K$-band spectrum implies that $T_{\text {eff }} \leq 34000 \mathrm{~K}$. Also shown are the theoretical evolutionary tracks and isochrones from Lejeune \& Schaerer (2001). Right: the same region of the HRD as shown in the left panel, but now including the regions in the HRD capable to produce $\mathrm{Q}_{0}$ and $\mathrm{Q}_{1}$ for comparison.

ionizations. We find that the number of Lyman continuum photons is $7.3 \pm 1.2 \times 10^{47}$. The given accuracies reflect the error in the distance quoted by Brown et al. (1994).

Krügel et al. (1982) performed observations of the radio recombination lines $\mathrm{H} 76 \alpha$ and $\mathrm{He} 76 \alpha$. The corresponding ionized helium fraction is $3 \%$, which leads to $2.2 \pm 0.1 \times$ $10^{46}$ helium continuum photons.

The observed number of photons capable of ionizing hydrogen $\left(\mathrm{Q}_{0}\right)$ and helium $\left(\mathrm{Q}_{1}\right)$ can be compared to predictions based on stellar atmosphere models of OB stars (Smith et al. 2002). If we assume that a single hot star ionizes NGC 2024, $\mathrm{Q}_{0}$ and $\mathrm{Q}_{1}$ constrain the location of the ionizing star in the HRD. In Fig. 4 (right panel), the regions which are able to produce the required amount of $\mathrm{Q}_{0}$ and $\mathrm{Q}_{1}$ are indicated by vertical and horizontal stripes, respectively. These regions overlap with the shaded region in the HRD defined by the spectral classification of IRS2b.

\section{Discussion}

As shown in the previous section, an O8 star with characteristics compatible with those observed in IRS2b should be capable of producing the required amount of ionizing radiation to explain the degree of ionization of NGC 2024. To meet the constraint set by $\mathrm{Q}_{1}$, its spectral type is more likely to be late $\mathrm{O}$ than early B, which is supported by its position in the CMD. Note, however, that the predicted value for $\mathrm{Q}_{1}$ is not well known and depends on the selected stellar model. Although other candidate ionizing stars exist, they are not needed to provide a self-consistent solution for the ionization of NGC 2024. In the following we will discuss some of these alternatives.

If we correct for foreground extinction (cf. Fig. 2), IRS2b and IRS2 are the two brightest sources. The position of IRS2 in the CMD indicates a strong infrared excess (Lenorzer et al. 2003; Grasdalen 1974). IRS2 is associated with the ultracompact radio source G206.543-16.347, but its physical nature is unclear. Current models suggest that ISR2 is a B star surrounded by a dense circumstellar disk responsible for the production of the infrared excess.

IRS1 (estimated spectral type B0.5V, Garrison 1968) and IRS2 have been candidate ionizing sources for a long time. Barnes et al. (1989) discovered 29 additional near-infrared sources in the Flame Nebula. In Figs. 1 and 2, the brightest of these infrared sources discovered by Barnes et al. (1989) are 
plotted, as well as IRS1 and IRS2. They propose that IRS1, IRS4 and IRS5, together with IRS3, provide a significant contribution to the ionizing radiation. IRS3 is not shown in the CMD, because it is not a single source, but consists of multiple stars which were not resolved by Barnes et al. (1989). $A s Q_{1}$ is decreasing very rapidly with later spectral type, the contribution to $\mathrm{Q}_{1}$ by the other bright stars (all later spectraltype than $\mathrm{B} 0.5 \mathrm{~V}$ ) is negligible.

The radio continuum emission, however, can only be transformed into the number of ionizing photons assuming that there is no dust included in the HII region, and that the H II region is ionization bounded. If these assumptions are not valid, the derived values of $\mathrm{Q}_{0}$ and $\mathrm{Q}_{1}$ are lower limits, so that a star hotter than IRS2b might be required. In principle, such a star could be significantly more reddened $\left(A_{V} \geq 35 \mathrm{mag}\right.$, based on our detection limit in $J$ ) and thus have remained undetected, especially when the amount of extinction strongly varies with position. However, the visual extinction we measure for the stars in the field is in the range between $5.5 \leq A_{V} \leq 25 \mathrm{mag}$. We judge that the probability of selectively obscuring this potentially hotter star is low.

In Fig. 4 evolutionary tracks and isochrones from Lejeune \& Schaerer (2001) are plotted and the zero-age main sequence (ZAMS) is indicated. The location of IRS2b suggests that its mass is between 15 and $25 M_{\odot}$. Although it is not possible to determine the age of a single star, the position of IRS2b in Fig. 4 is, given the uncertainty in the effective temperature calibration consistent with the age proposed by Blaauw (1991), Brown et al. (1994) and Comerón et al. (1996). Comerón et al. (1996) derive an age of $2 \times 10^{6}$ years based on the frequency of IR excess in very low mass objects in NGC 2024 as compared to that in Rho Ophiuchi.

Acknowledgements. $\mathrm{AB}$ acknowledges financial support from the DFG during a two-month visit at ESO Headquaters. LK is supported by a fellowship of the Royal Academy of Arts and Sciences in The Netherlands. We would like to thank L. Decin for her help in the data reduction of the $L$ band spectrum. We thank the anonymous referee for critical and constructive comments. NSO/Kitt Peak FTS data used here were produced by NSF/NOAO.

\section{References}

Aller, L. H., Appenzeller, I., \& Baschek, B. E. A. (eds.) 1982, Landolt-Bornstein: Numerical Data and Functional Relationships in Science and Technology

Barnes, P. J., Crutcher, R. M., Bieging, J. H., Storey, J. W. V., \& Willner, S. P. 1989, ApJ, 342, 883

Bik, A., Kaper, L., Hanson, M. M., \& Comerón, F. 2003, A\&A, submitted

Blaauw, A. 1991, in NATO ASIC Proc. 342, The Physics of Star Formation and Early Stellar Evolution, 125

Brown, A. G. A., de Geus, E. J., \& de Zeeuw, P. T. 1994, A\&A, 289, 101

Cardelli, J. A., Clayton, G. C., \& Mathis, J. S. 1989, ApJ, 345, 245

Comerón, F., Rieke, G. H., \& Rieke, M. J. 1996, ApJ, 473, 294

Garrison, R. F. 1968, PASP, 80, 20

Grasdalen, G. L. 1974, ApJ, 193, 373

Haisch, K. E., Lada, E. A., Piña, R. K., Telesco, C. M., \& Lada, C. J. 2001, AJ, 121, 1512

Hanson, M. M., Conti, P. S., \& Rieke, M. J. 1996, ApJS, 107, 281

Jiang, D. R., Perrier, C., \& Lena, P. 1984, A\&A, 135, 249

Kaper, L., Bik, A., Comerón, F., \& Hanson, M. M. 2003, A\&A, submitted

Koornneef, J. 1983, A\&A, 128, 84

Krügel, E., Thum, C., Pankonin, V., \& Martin-Pintado, J. 1982, A\&AS, 48, 345

Lada, E. A., Evans, N. J., Depoy, D. L., \& Gatley, I. 1991, ApJ, 371, 171

Lee, T. A. 1968 , ApJ, 152, 913

Lejeune, T., \& Schaerer, D. 2001, A\&A, 366, 538

Lenorzer, A., Bik, A., de Koter, A., et al. 2003, A\&A, submitted

Lenorzer, A., Vandenbussche, B., Morris, P., et al. 2002, A\&A, 384, 473

Martins, F., Schaerer, D., \& Hillier, D. J. 2002, A\&A, 382, 999

Nisini, B., Smith, H. A., Fischer, J., \& Geballe, T. R. 1994, A\&A, 290, 463

O'dell, C. R. 2001, ARA\&A, 39, 99

Smith, L. J., Norris, R. P. F., \& Crowther, P. A. 2002, MNRAS, 337, 1309

Stetson, P. B. 1987, PASP, 99, 191

Vacca, W. D., Garmany, C. D., \& Shull, J. M. 1996, ApJ, 460, 914 